\title{
PERSUASIVE STRATEGIEN AUF DEN INTERNETSEITEN FÜR VERHÜTUNGSMITTEL
}

\begin{abstract}
Persuasive strategies on websites about contraception
The article discusses typical persuasive strategies used by leading producers of contraceptives on their websites and on the websites of particular contraceptive products from the media-linguistic point of view. The purpose of the paper is to examine illocutions, text-clusters and the text-image relation through which the author wants to persuade about the quality of his products and to present arguments for using hormonal contraception. The article also shows which strategies are used to advertise the pill as a lifestyle and beauty product.
\end{abstract}

KEYWORDS: discourse, contraception, text-cluster, persuasion

In Deutschland ist die Pille das Verhütungsmittel Nummer eins. Rund 70 Anti-Baby-Pillen sind derzeit auf dem Markt und ca. 60 Prozent aller geschlechtsreifen Frauen nehmen die Pille. Laut einer Studie der Bundeszentrale für gesundheitliche Aufklärung von $2011^{1}$ nehmen insgesamt $72 \%$ der 20- bis 29-Jährigen die Pille ein. Bei den 20- bis 44-Jährigen sind es 55\%. Weltweit verhüten 120 Millionen Frauen mit hormonellen Tabletten und der weltweite Umsatz der Pille beträgt ca. 9,3 Mrd. Dollar. Alleine in Deutschland sind das 577 Millionen Euro jährlich.

Seit vielen Jahren wird die Pille zum Symbol sexueller Befreiung stilisiert (Staupe \& Vieth, 1996, S. 127). Für viele Frauen gehört die Pille einfach zum Leben dazu. Zum Leben gehört auch die Werbung für die Pille und der Kampf um die Kundinnen und um ihre Überzeugungen. Die Konkurrenz auf dem Markt ist groß und die Pharmaunternehmen suchen nach neuen Absatzmöglichkeiten, Zielgruppen und Werbestrategien. Eine neue Zielgruppe sind die jungen Mädchen, die den Absatz der Pille steigern könnten. Die Analyse der Werbung der Pharmakonzerne zeigt, dass die Pille zunehmend auch als Lifestyleprodukt propagiert wird. Auf diesem boomenden Markt spielt heute die Einnahme von als schönheitsfördernd beworbenen hormonellen Kont-

\footnotetext{
Marcin Maciejewski - Uniwersytet im. Adama Mickiewicza, Poznań, marcys@amu.edu.pl

${ }^{1}$ Bundeszentrale für gesundheitliche Aufklärung: Verhütungsverhalten Erwachsener - Ergebnisse der Repräsentativbefragung, 2011.
} 
razeptiva eine wesentliche Rolle. Für die Verhütungsmittel mit klangvollen Namen wie Belara, Yasminelle, Pink Luna und Petitbelle und für den damit verbundenen Lebensstil wird intensiv geworben. Nach Schindele (2012, S. 6) schlucken viele Frauen nicht in erster Linie zur Verhütung einer ungewollten Schwangerschaft die Pille, sondern auch zur Verschönerung von Haut und Haaren, zur Kontrolle über den Zeitpunkt der Monatsblutung und zur Minimierung körperlicher Beschwerden vor der Menstruation. Die Pharmakonzerne werben mit der Botschaft, dass Frauen über ihre Sexualität und ihr sexuelles Leben die volle Kontrolle haben und sie beliebig regulieren können (vgl. Duden, 1996, S. 69).

Durch die zunehmende Verbreitung elektronischer Medien hat sich der Markt auf das World Wide Web verlagert, wo die Pharmakonzerne die hormonellen Kontrazeptiva bewerben. Das Internet ist heutzutage für junge Frauen eine wichtige Informationsquelle zum Thema Sexualität und Verhütung ${ }^{2}$. Einen wichtigen Hintergrund für das kommunikative Handeln der Pharmaunternehmen bilden die werberechtlichen Faktoren. Den wichtigsten rechtlichen Rahmen stellt das Heilmittelwerbegesetz (HWG) dar. Es regelt das Werben um Medikamente und Informieren der Patienten. Nach dem Heilmittelwerbegesetz dürfen bestimmte Informationen nur medizinischen Fachkreisen zur Verfügung gestellt werden. Die Laien-Werbung $($ DTC $=$ Direct To Consumer) für rezeptpflichtige Medikamente ist verboten ${ }^{3}$. Einen sehr wichtigen Teil des Kommunikationskontextes bildet der soziokulturelle Aspekt der Nutzung von Verhütungsmitteln, die auch Elemente der Diskursstränge verschiedener Diskurse sind. Sie kommen in den Diskursen zum Thema Sexualerziehung, Sexualpädagogik und Präventionskampagnen mit den Themen Aids, sexuelle Gewalt, Armut, Feminismus und Selbstbestimmung der Frau vor. Besonders die aktuellen Trends (z. B. Sexualisierung der Kultur und der Lifestylemuster, Pluralität der Wertvorstellungen) stehen im Wechselspiel zwischen sozialen und gesamtpsychologischen Veränderungen der Gesellschaft. Darauf reagiert auch die Werbung, die man als Spiegel unserer Alltagskultur betrachten kann (vgl. Karmasin, 2001, S. 213). Auch die Werbung für Verhütungsmittel lässt auf das heutige Wertesystem und Trends im sexuellen Verhalten (junger) Menschen schließen. Die Werbung für Verhütungsmittel schöpft aus diesem Wertesystem und Pharmakonzerne sprechen die Zielgruppen mit kollektiven Vorstellungen von Werten an.

\footnotetext{
${ }^{2}$ Repräsentativbefragung zur Jugendsexualität der Bundeszentrale für gesundheitliche Aufklärung: Jugendsexualität im Internetzeitalter, 2013.

${ }^{3}$ Derzeit wird in der Europäischen Union über die möglichen Folgen des Aufhebens dieses Werbeverbots diskutiert. Die Gegner solcher Lösungen führen das Beispiel der USA an, wo seit über 20 Jahren massiv für rezeptpflichtige Medikamente geworben wird und wo die Auswirkungen von Direktwerbung für rezeptpflichtige Arzneimittel (DTC) auf Verschreiber und Patienten sichtbar sind. Als Resultat der uneingeschränkten Werbung medizinischer rezeptpflichtiger Produkte werden unvollständige und irreführende Information, der kräftig steigende Umsatz beworbener Medikamente und häufige Wunschverschreibungen genannt (Diekwisch \& Schaaber, 2009, S. 329; Kravitz \& Epstein et al., 2005, S. 1999).
} 


\section{Ziele und Korpus}

Der Beitrag situiert sich im Kontext einer pragmatisch orientierten Medienlinguistik. Es wird das Ziel verfolgt, aus der medienlinguistischen Perspektive die typischen persuasiven Handlungen auf den Webseiten von Pharmaunternehmen und Webseiten konkreter Verhütungsmittel zu ermitteln. Um die volle Bandbreite des persuasiven Potentials aufzuzeigen, werden sowohl sprachliche als auch multimediale Elemente mit einbezogen. Eine Webseite wird hier als semiotisch komplexer Supertext betrachtet, der aus dem sprachlichen Text und den visuellen Elementen (visuellen Texten) besteht (vgl. Fix, 1996, S. 116). Persuasives Handeln wird verstanden als Versuch, andere mit sprachlichen und nichtsprachlichen Mitteln dazu zu bringen, die eigene Perspektive auf einen in Frage stehenden Sachverhalt zu verändern bzw. anhand der Information über einen schon bewerteten Gegenstand erst eine dementsprechende Perspektive zu gewinnen (vgl. Herbig \& Sandig, 1994, S. 61).

Im Internet gibt es einige Typen von Webseiten, die ausschließlich dem Thema Verhütung gewidmet sind. Sie lassen sich im Allgemeinen in Webseiten, die von öffentlichen oder kommerziellen Institutionen betrieben werden, einteilen. $\mathrm{Zu}$ den ersten gehören z. B. die Bundeszentrale für gesundheitliche Aufklärung (familienplanung. de) und Profamilia (profamilia.de) ${ }^{4}$. Die kommerziellen Webseiten lassen sich in drei Gruppen klassifizieren. Die erste bilden Webangebote von Pharmaunternehmen, die in Form einer Rubrik vorkommen (z. B. jenapharm.de/verhuetung). Sie werden auch mit anderen Adressen (hauptsächlich nicht aktiven Webseiten konkreter Präparate) verlinkt. Zu der zweiten Gruppe gehören Internetseiten konkreter medizinischer Produkte (z. B. Ellaone, Vikela). Auf diesen Produktwebseiten ist der Betreiber einfach identifizierbar (z. B. durch das Logo und/oder den Namen des Herstellers). Die dritte Gruppe umfasst Webseiten, die nicht einem Präparat, sondern allgemein den Verhütungsmitteln oder einer Verhütungsmethode gewidmet sind und auf denen der Betreiber nur indirekt $\mathrm{zu}$ ermitteln ist. In diesem Beitrag interessieren uns jedoch nur Webseiten kommerzieller Organisationen, welche zur Absatzförderung eigene hormonelle Kontrazeptiva auf dem Markt haben, die sie bewerben und dabei Vermarktungsinteressen verfolgen.

Als Grundlage der Untersuchung dient ein Korpus, das sich aus Webseiten von Pharmakonzernen und Webseiten konkreter medizinischer Produkte zusammensetzt. Die Webseiten, auf denen die hormonellen Kontrazeptiva beworben werden, wurden in dem Zeitraum von Mai 2015 bis Oktober 2015 untersucht. Es wurden 17 Webseiten analysiert. Fünf davon sind Webseiten von konkreten Verhütungsmitteln, sechs sind feste Rubriken von Pharmaunternehmen zum Thema Verhütung und sechs sind allgemeine Portale zum Thema Verhütung, die von Pharmaunternehmen betrieben werden

\footnotetext{
${ }^{4}$ Dem Thema Verhütung werden auch umfangsreiche Rubriken auf allgemeinen Gesundheitsportalen gewidmet (z. B. omeda.de, gofeminin.de), auf denen Werbebanner der Pharmakonzerne veröffentlicht werden. Es gibt aber auch Webseiten über Verhütung, bei denen nicht klar zu erkennen ist, wer sie betreibt.
} 
(z. B. www.gynaekologie.hexal.de, www.meineverhütung.ch, www.schoen-sicher.de, www.mädchensprechstunde.de).

Die methodologische Umsetzung des Forschungsvorhabens beruht vor allem auf der Identifizierung unterschiedlicher Text-Cluster auf den Webseiten, die nach Püschel (1997, S. 38) fest miteinander gekoppelte Strukturelemente der Webseiten (textuelle und graphische Module) bedeuten. Die Text-Cluster werden hier also als eine multimodale Einheit, als multimodale Verknüpfung (Klug \& Stöckl, 2016, S. VII) verstanden, die sowohl als ein Hypertextmodul (z. B. eine Text-Bild-Einheit mit einer Hyperlinkfunktion oder eine verlinkte PDF-Datei zum Herunterladen), als auch als eine einfache Verbindung verbaler und nonverbaler Elemente auf einer Webseite auftreten kann.

Es ist charakteristisch, dass die analysierten Webseiten eine relativ einfache modulare Struktur haben und die Text-Cluster (vor allem auf den Startseiten) ihre grundsätzlichen Bausteine bilden. Diese Module stellen die graphisch markierten Bereiche einer Webseite dar. Die Text-Cluster bilden nicht nur aus dem Grund eine Einheit, weil sie strukturell als Text-Bild-Elemente erscheinen und dadurch schnell zu identifizieren sind. Die Verbindung von Text und Bild bildet in den meisten Fällen ein Text-Cluster, das zugleich als Hyperlink fungiert. Somit erfüllen sie auch eine Navigationsfunktion und gehören zu der Gruppe der interaktiven Module.

\section{Persuasive Text-Cluster}

Den strukturellen Hintergrund für die persuasiven Handlungen in Text-Clustern bildet der übersichtliche Aufbau der Webseiten. Durch ein nicht überladenes Layout kann die Aufmerksamkeit der Leser leicht auf die Text-Cluster gelenkt werden. Die Autoren statten viele Text-Cluster auf der Homepage mit hypermedialen Eigenschaften aus, stellen programmierte Beziehungen zwischen bestimmten Hypertextmodulen her, um im Navigationsmodus die Internetnutzer zu beeinflussen. Die Text-Cluster dienen in der Folge als Einladung zum Weiterklicken. Die geplante Persuasivität ergibt sich außerdem aus ihrer Platzierung im zentralen Bereich auf der Startseite und auf weiteren mit ihr verlinkten Webseiten. Auch das häufige Vorkommen der persuasiven Text-Cluster auf verschiedenen Homepages von Unternehmen und innerhalb ihrer Strukturen zeugt davon, dass ihre Hervorhebung eine gezielte persuasive Maßnahme ist.

Die Identifikation der Text-Cluster im Material erfolgt einerseits anhand der strukturellen Faktoren, der Verbindung sprachlicher Mittel mit Nonverbalem (gekoppelte Text-Bild-Module) und andererseits anhand ihrer dominierenden Sprechhandlung. Die Analyse ergibt, dass es auf allen Webseiten drei grundlegende Gruppen von Text-Clustern gibt. Jedem Text-Cluster liegt eine paraphrasierte Formel zugrunde, die die persuasiven Intentionen der Autoren offenlegt und die man handlungstheoretisch folgendermaßen ausdrücken kann: Der Autor wirbt für die Pille, indem er die Anwendung 
der Pille mit Schönheit (Text-Cluster Pille und Schönheit), mit Lifestyle (Text-Cluster Pille und Lifestyle) und mit Sicherheit (Text-Cluster Pille und Sicherheit) koppelt.

Die Bezeichnungen der Text-Cluster knüpfen also an die Intention des Autors an, sie werden aber zugleich durch die grundlegenden Schlüsselwörter, die in diesen Text-Clustern aber auch im Pillen-Diskurs vorkommen, charakterisiert. Diese strikte Trennung ist eher analytischer Natur. Die Autoren können in einem Text-Cluster auch andere illokutionäre Ziele realisieren (z. B. in dem Text-Cluster Pille und Schönheit können Unternehmen auch die Sicherheit der Pille anpreisen).

Im Folgenden werden die identifizierten Text-Cluster hinsichtlich ihrer persuasiven Eigenschaften charakterisiert. Es wird auf ihre wichtigsten Sprechhandlungen ${ }^{5}$, auf die nonverbalen Elemente und verwendete Schlüsselwörter eingegangen. Um die visuelle Ebene der Text-Cluster zu interpretieren, werden konkrete Beispiele der Begleittexte angeführt, die für das Verstehen des Bildes wichtig sind.

a) Text-Cluster Pille und Schönheit (Persuasive Handlung: Kopplung der Wirkungsweise hormoneller Verhütungsmittel mit Schönheit)

Ein konventionelles persuasives Mittel der Pharmaunternehmen ist die Kopplung der Wirkungsweise hormoneller Verhütungsmittel mit der Schönheit. Diese Handlung wird in zwei parallelen illokutiven Schritten realisiert. In dem ersten bewerten die Unternehmen die Schönheit und verleihen ihr einen sehr hohen Status. Im zweiten stellen sie einen Zusammenhang zwischen der Schönheit und der Anwendung der Pille fest. Auf den Webseiten werden in den Text-Clustern auch viele Schönheitstipps und Themen rund um das Aussehen angesprochen und dabei wird die antiandrogene Wirkung der Hormonpräparate betont.

Du bist schön! Damit du dich auch immer wohl fühlst, haben wir dir die wichtigsten Tipps und Grundlagen rund um die Themen Body, Beauty \& Klamotten zusammengestellt (Dr. Kade www. schoen-sicher.de).

Ein weiterer Vorteil ist der sog. antiandrogene (anti: gegen, androgen: männlich) Effekt von einigen in Pillen enthaltenen Gestagenen, z. B. Chlormadinonacetat oder Dienogest. Dieser kommt gerade jungen Mädchen mit Akne aber auch Frauen mit fettiger, zu Unreinheiten neigender Haut und leicht fettenden Haaren zu Gute, da diese Erscheinungen auf einen Überschuss an männlichen Hormonen, den Androgenen, zurückzuführen sind. Durch die Gabe der oben genannten Gestagene kann dem entgegengewirkt werden (Dr. Kade, www.schoen-sicher.de/faq/wirkung/weitere-vorteile-der-pille).

(3) Häufig leiden gerade Mädchen und junge Frauen unter Hautunreinheiten oder Akne. Diese beruhen größten Teils auf einem Überschuss an männlichen Hormonen (Testosteron), die für eine übermäßige Talgproduktion verantwortlich sind (androgener Effekt). Einige Gestagene, die in den Mikropillen enthalten sind, wirken diesem sog. androgenen Effekt entgegen und haben somit einen vorteilhaften Effekt auf das Hautbild (Dr. Kade, www.schoen-sicher.de/die-pille/was-kanndie-pille-noch).

${ }^{5}$ Nach Rolf werden Sprechhandlungen als Realisierungen von Funktionen sprachlicher Zeichen verstanden (vgl. Rolf, 1997, S. 124). 
In dem Text-Cluster „Information für Anwenderinnen zum Herunterladen“ über das Verhütungsmittel Belara (siehe Abbildung Nr. 1) wird verbal und nonverbal ein enger Zusammenhang zwischen Pille und Schönheit (ausgedrückt durch eine Rose) suggeriert.

\begin{abstract}
Das Besondere an Belara ist das Gestagen Chlormadinonacetat und seine zusätzliche Wirkung auf die Haut: Es wirkt dem Einfluss von Androgenen (männlichen Hormonen) entgegen, die Ursache für unreine Haut und fettiges Haar sein können. So wirkt Belara empfängnisverhütend und kann gleichzeitig das Erscheinungsbild von Haut und Haaren verbessern (www.grunenthal. de/cms/cda/_common/inc/display_file.jsp?fileID=33300217).
\end{abstract}

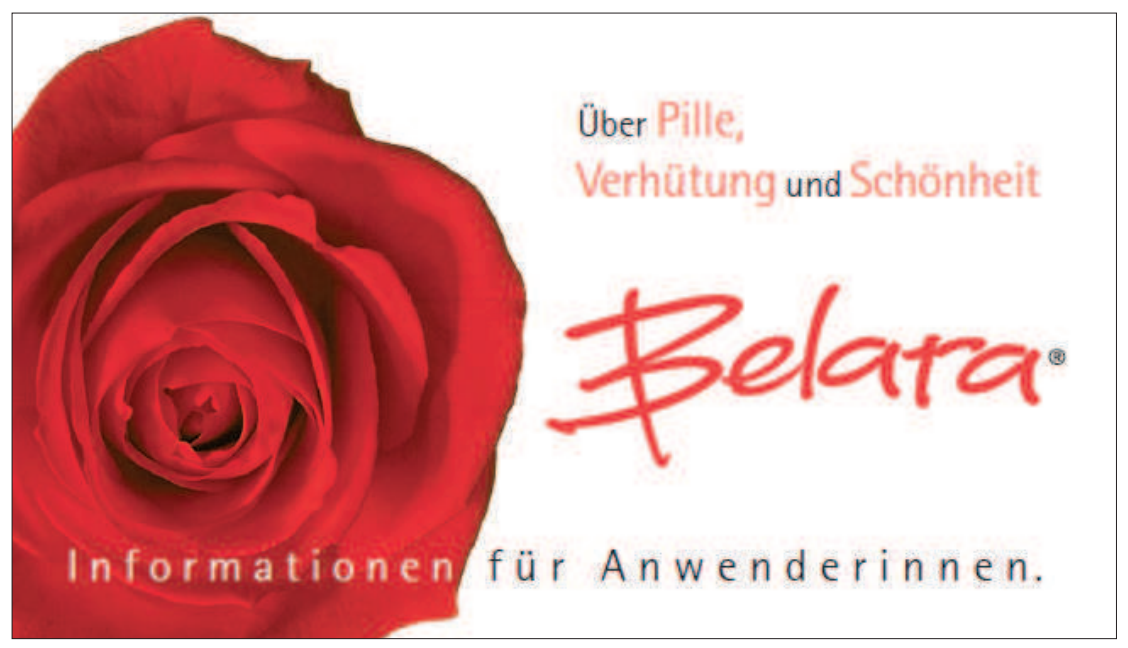

Abb. 1: Ein Text-Cluster über die Pille Belara (www.grunenthal.de/cms/cda/_common/ inc/ display_file.jsp?fileID=33300217)

Die Leserinnen können auf dieser Website lesen, wie sie „mit der Pille das Gewicht halten“ können. Moderne Pillen könnten „Wassereinlagerungen und ihren Begleiterscheinungen entgegenwirken“. Die Website weist Mädchen, die sich in der Pubertät befinden, darauf hin, dass die Pille auch gegen Pickel wirke:

Haut und Haare werden schöner, und du kannst im Spiegelbild wieder deinem strahlenden Ebenbild begegnen. [...] Antiandrogene Pillen sind das Plus in jeder Empfängnisverhütung. Mit ihnen kannst du nicht nur zuverlässig verhüten, du kannst auch etwas für Haut und Haare und damit fürs Aussehen tun (Jenapharm, zit. nach Blech, 2013, S. 114).

Das Wort Schönheit ist das am meisten verwendete Schlüsselwort in den Clustern aus dieser Gruppe. Es kommt sogar in der Webadresse des Pharmaunternehmens Dr. Kade vor. Gleich auf der Homepage sehen wir zwei Flashbanner, in denen das Wort Schönheit dominiert. Der Sender betont, dass die Empfängerinnen schön seien. Auf der nonverbalen Ebene werden Bilder von schönen Frauen und schönen Gesichtern präsentiert.

b) Text-Cluster Pille und Lifestyle (Persuasive Handlung: Kopplung der Wirkungsweise hormoneller Verhütungsmittel mit Lifestyle) 
Durch Texte und Bilder in diesen Text-Clustern akzentuieren und bewerten die Autoren vor allem den modernen Lifestyle, für den Freiheit, Unbeschwertheit, Freude und Zufriedenheit charakteristisch sind und der aus der Wahl der richtigen Verhütungsmethode resultieren soll. In den Text-Clustern sehen wir auf der nonverbalen Ebene Bilder zufriedener Paare und Frauen, die Freude im Zusammenhang mit einem Verhütungsmittel zeigen (siehe Abbildung Nr. 2).

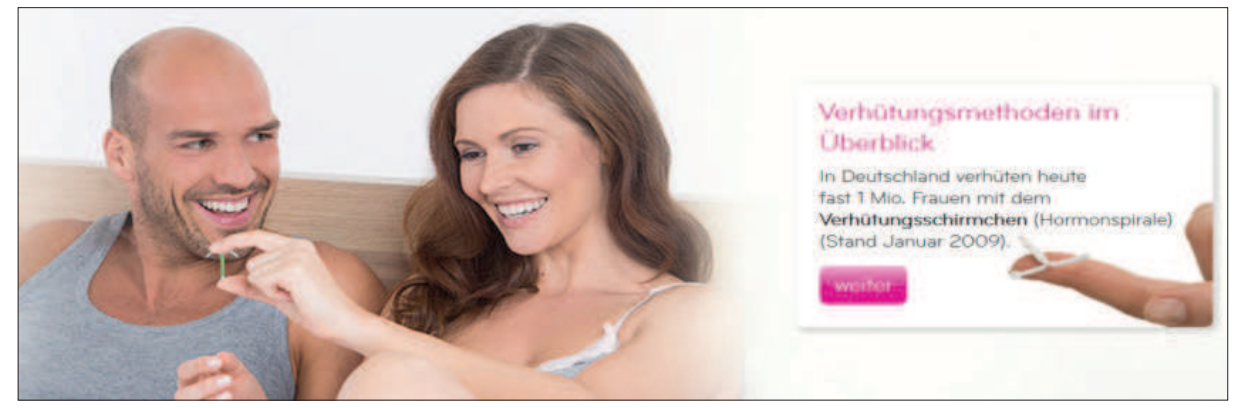

Abb. 2: Text-Cluster Verhütungsmethoden im Überblick auf der Homepage von Jenapharm (www.jenapharm.de/verhuetung/alternativen-zur-pille/)

Der moderne, angestrebte Lifestyle wird in diesen Text-Clustern fast glorifiziert und durch weitere wichtige Schlüsselwörter wie Selbstbestimmung, Unabhängigkeit (u. a. im Vergleich zu den früheren Zeiten) charakterisiert. Dieser Lifestyle wird positiv bewertet und es wird nahe gelegt, dass es ausreichend viele wichtige Gründe gibt, sich für Verhütungsmethoden, die diesen Lifestyle ermöglichen, zu interessieren (siehe Abbildung Nr. 3). Auf dem Bild sieht man eine junge Frau, die froh zu sein scheint, dass sie noch viele Pläne vor sich hat, welche sie selbstbestimmt realisieren kann, ohne auf das Sexualleben verzichten und an ein Kind denken zu müssen.

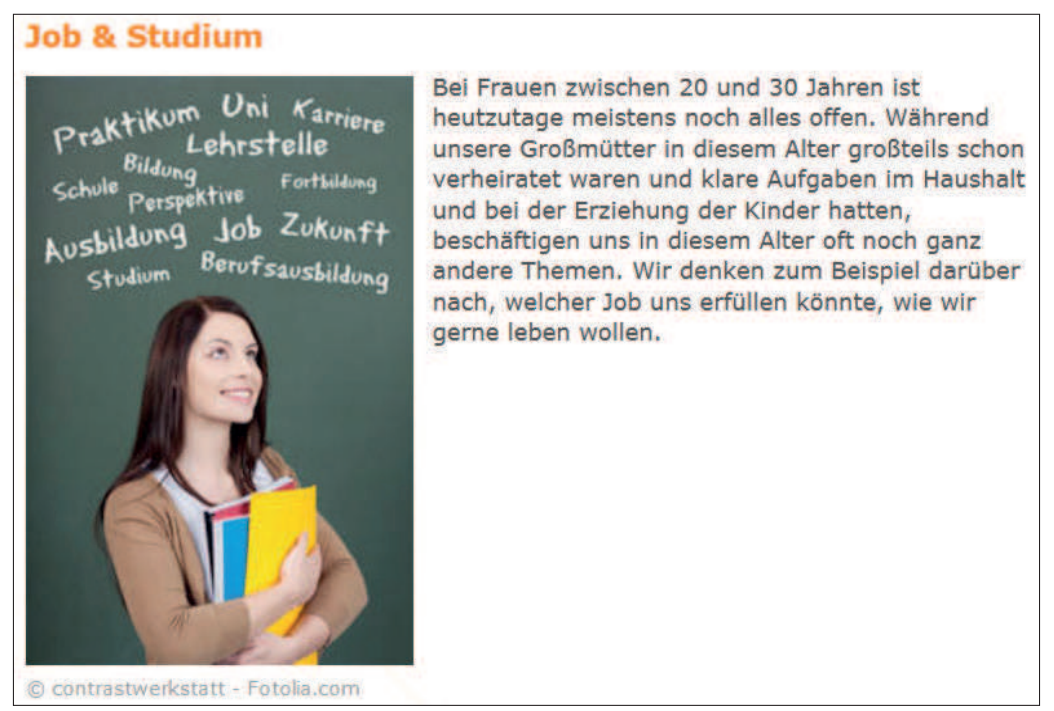

Abb. 3: Text-Cluster Job und Studium auf der Homepage von Jenapharm (www.jenapharm.de/verhuetung/alternativen-zur-pille/) 
Die Selbstbestimmung und Unabhängigkeit wird bei Jenapharm mittels Web-Logo des Konzerns ausgedrückt.

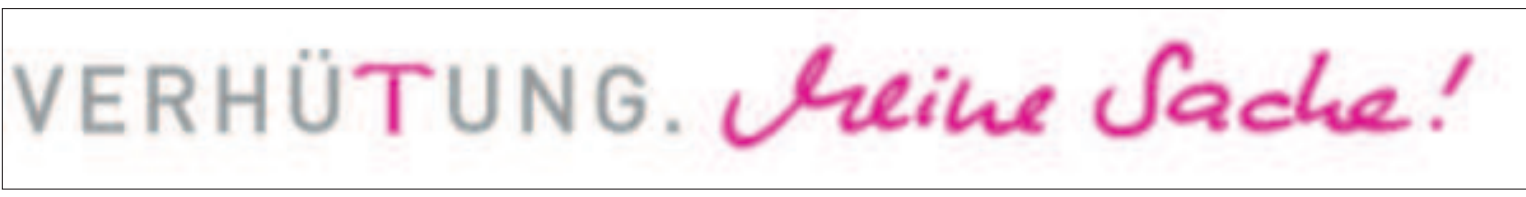

Die Pharmaunternehmen stellen in den meistens textdominanten Text-Clustern auf ihren thematischen Webseiten über Verhütung das Schicksal verschiedener Frauen dar, beschreiben ihren Lebensstil, Probleme, die von den Leserinnen nachzuvollziehen sind. Mit dieser persuasiven Strategie zeigen die Pharmaunternehmen, dass sie die Situation, Bedürfnisse und Probleme der Frauen verstehen und sich in ihre Lage versetzen können. Das illokutive Ziel dabei lässt sich leicht ermitteln: Verständnis gegenüber Frauen zeigen und das als Grundlage weiterer Überzeugungsarbeit nutzen. Die Frauen werden damit direkt angesprochen (z. B. mittels Schlüsselwörtern wie Selbstverwirklichung, Freiheit, Unabhängigkeit, sorgloses Leben, Erfolg) und finden in diesen Beschreibungen Elemente ihrer Persönlichkeit. Sehr deutlich ist das auf der Seite www.pillen-fragen. de der Firma Gedeon Richter Pharma sichtbar. Der Sender stellt die Situationen dreier Frauen dar, indem er drei grundlegende Phasen im Leben einer Frau (Pubertät, Phase der Ausbildung, Beruf) widerspiegelt.

Mittendrin in der Wunderwelt

In der Pubertät fahren die Gefühle manchmal Achterbahn. Du bist mittendrin - in dieser Wunderwelt - und gerade dabei, spannende Dinge zu entdecken... Klick dich einfach durch (Gedeon Richter Pharma, www.pillen-fragen.de).

\section{(7) Einfach fabelhaft!}

Ein schönes Gefühl, wenn einem die ganze Welt offen steht! Irrungen und Wirrungen der Teenagerzeit liegen weit hinter Ihnen. Sie stehen mit beiden Beinen im Leben und sind unabhängig. Deswegen haben Sie auch viel Zeit, sich mit sich selbst zu beschäftigen - neben Aussehen und Wohlbefinden spielt für Sie Selbstverwirklichung eine große Rolle.

Viel Freiheit bedeutet nur manchmal auch Unsicherheit. Sicher gibt es Momente, in denen auch Sie gerne irgendwo ankommen und sich langfristig für einen Weg entscheiden möchten - sowohl privat als beruflich. Nur verständlich, wenn Sie manchmal von Zukunftsängsten geplagt werden. Es ist nicht immer leicht, die Freiheit auch genießen zu können (Gedeon Richter Pharma, www.pillen-fragen.de).

(8) Lisas Lächeln steckt an.

Sind Sie manchmal froh, keine 20 mehr zu sein? Wie angenehm, endlich den richtigen Job und den passenden Partner zu haben und anspruchsvoll und kritisch Entscheidungen zu treffen. Weil Sie einfach wissen, was Ihnen gut tut und wichtig ist. Manchmal ist es aber schwierig, dabei die Balance zu halten: Sie wollen Erfolg im Job und Zeit für sich. Ihre Beziehung ist Ihnen wichtigaber auch Ihre Freiheit. Sie möchten gesund leben und gleichzeitig sorglos sein. Sie wollen sich fühlen wie die Göttin, die Sie sind (Gedeon Richter Pharma, www.pillen-fragen.de). 
In diesen Clustern sieht man deutlich die Bemühungen des Senders, einige wichtige Begriffe zu modifizieren. Das Ziel der Modifikation der Begriffe ist die Bestätigung des von den Pharmakonzernen angestrebten und propagierten Lifestyles. Durch ihre Veränderung wird die Vermittlung der persuasiven Botschaft und der diskursiven Positionen des Senders begünstigt. Die modifizierenden Handlungen werden auf unterschiedliche Hypertextknoten verteilt (z. B. in den Text-Clustern auf der Homepage, FAQ-Rubriken usw.).

Die Modifikation der Begriffe umfasst in diesen Text-Clustern die Änderungen und Erweiterungen allgemeiner Begriffe wie Liebe und Verantwortung. Sie haben zum Ziel, bei den Empfängerinnen neue assoziative Verbindungen herzustellen. Eine neue Interpretation der Begriffe wird suggeriert. Die Leserinnen sollten beispielsweise das Wort Liebe mit Verhütung in Verbindung setzen. Es wird nahegelegt, dass bei Verliebtheit das Thema Verhütung zwingend zu berücksichtigen ist. Verhütung wird außerdem mit der ersten Liebe, mit dem ersten Mal, mit Schönsein, Frausein und Verliebtheit in Verbindung gebracht. Auf der Homepage der Firma Mibe (pille-fuer-mich.de) lesen wir nach dem Begrüßungsmodul:

(9) Wird aus der Schwärmerei mehr, kommen zum Kribbeln im Bauch und den glänzenden Augen oft noch andere Empfindungen, nämlich Lust und Leidenschaft. Und genau jetzt ist der richtige Zeitpunkt, eine kurze Pause einzulegen und nachzudenken (Mibe, www.pille-fuer-mich.de/Erstanwenderinnen/).

(10) Ein Widerspruch? Nein, denn Sex ist eigentlich nur dann schön und romantisch, wenn man dabei die Welt rund um sich vergessen kann - und keine Angst vor einer ungeplanten Schwangerschaft haben muss (Mibe, www.pille-fuer-mich.de/Erstanwenderinnen/).

(11) Deswegen gilt im Rausch der Gefühle: nicht ganz den Kopf verlieren, sondern vor dem Miteinanderschlafen darüber nachdenken, welche Verhütungsmethode am besten zu dir und deinem Partner passt (Mibe, www.pille-fuer-mich.de/Erstanwenderinnen/).

Charakteristisch ist der Spruch auf der Webseite von Jenapharm, der eine vom Sender beabsichtigte Assoziation schaffen sollte: „Weil Liebe und Verhütung zusammengehören“" (siehe Abbildung Nr. 4).

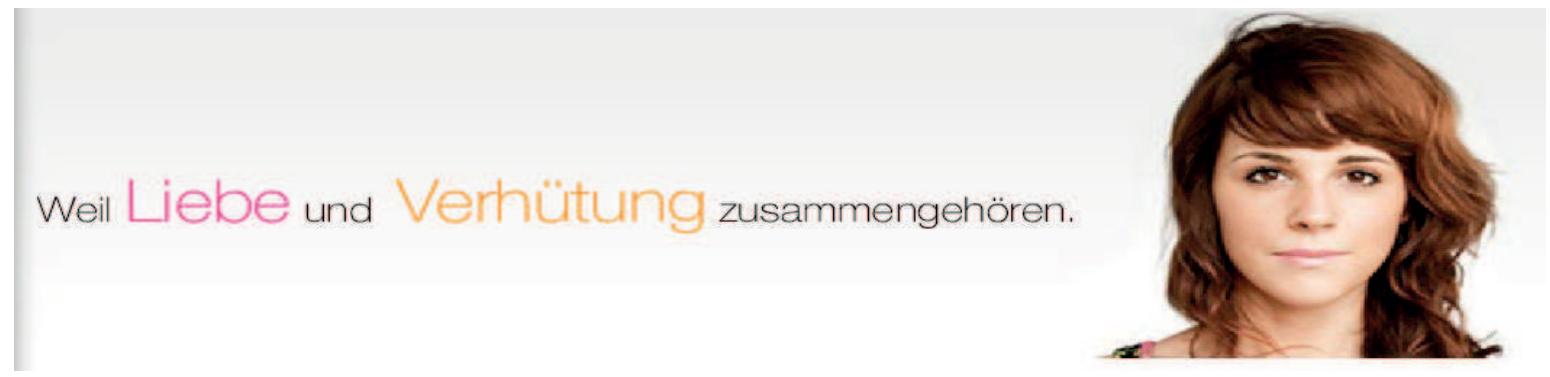

Abb. 4: Text-Cluster auf der Homepage von Jenapharm (www.jenapharm.de) 
Die Romantik, das Welt-Vergessen, Freiheit, ein Leben ohne Sorgen, die zum Lifestyle gehören, werden in den Text-Clustern nonverbal betont (siehe Abbildung Nr. 5).
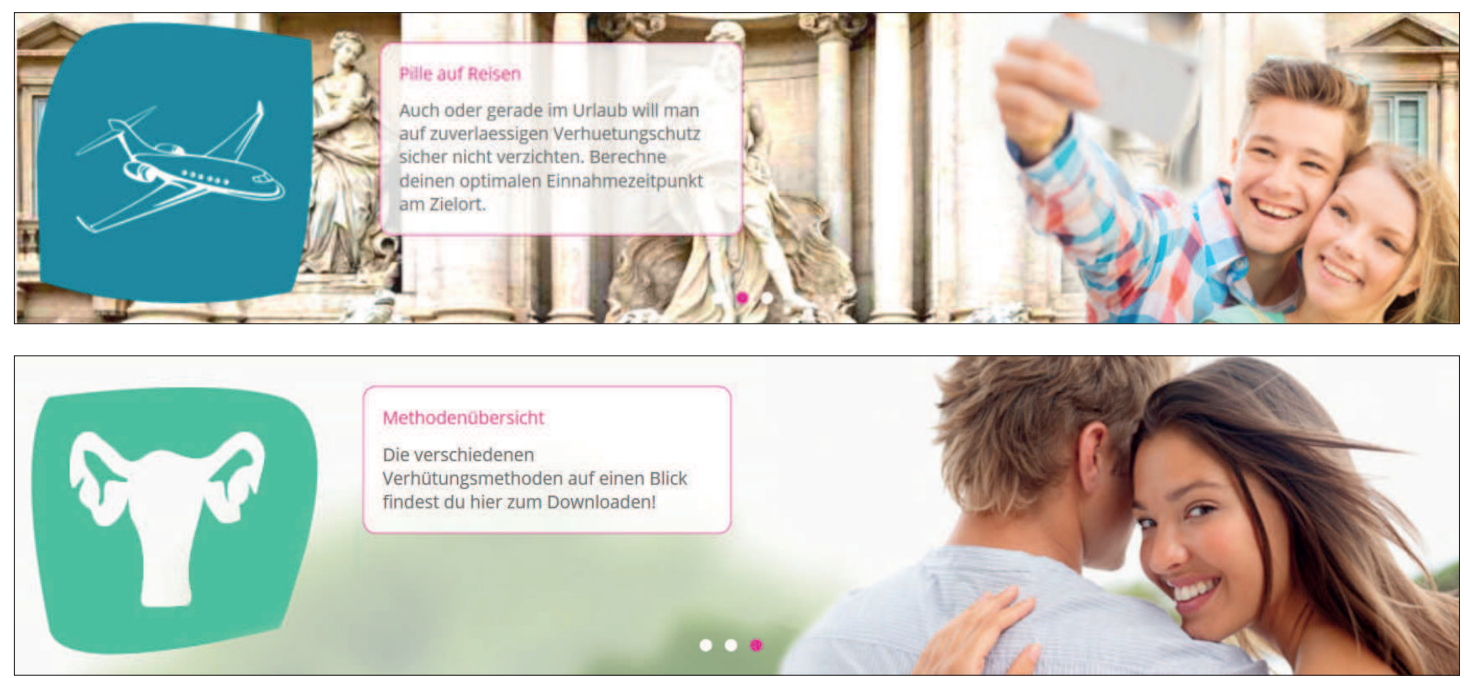

Abb. 5: Text-Cluster auf der Homepage (www.verhuetungsinfo.ch/de/hormonelle-verhuetungsmethoden, Bayer)

Die enge Beziehung zwischen Liebe und Verhütung legt auch der Titel der Homepage pillen-fragen.de der Firma Gedeon Richter nahe: „Wie Frauen lieben und verhüten". Er erscheint auf jeder Seite dieses Webangebots und wird im oberen Bereich als Logo in Textform platziert.

Eine Strategie, die hormonellen Verhütungsmittel in den Text-Clustern zu bewerben, ist es, die nicht-kontrazeptiven Wirkungen zu akzentuieren.

\section{Müssen Frauen wirklich bluten?}

Es gibt viele Mythen rund um den weiblichen Zyklus.

Erfahren Sie hier, was stimmt und was nicht. w mehr

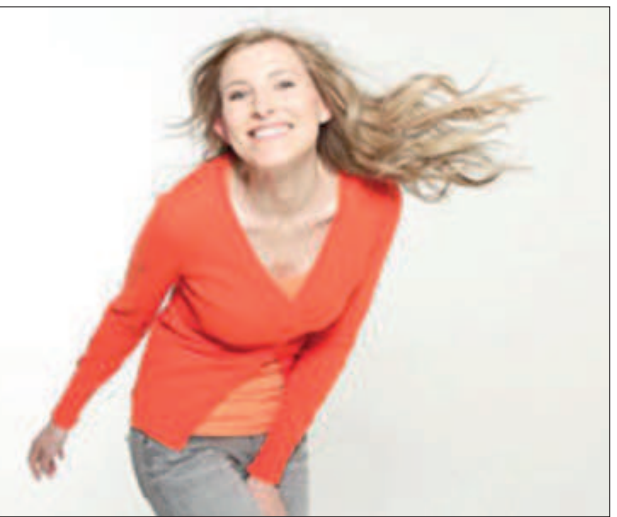

Abb. 6: Text-Cluster Müssen Frauen wirklich bluten? auf der Homepage von Jenapharm (www.meine-verhuetung.de)

Die Rolle der Verhütungsmittel wird in der Therapie starker Monatsblutungen exponiert. Monatsblutungen werden als etwas Lästiges, als überflüssig und etwas, das man vermeiden kann, dargestellt. Das lächelnde Gesicht der Frau auf der Abbildung 
6 suggeriert, dass die im Text-Cluster gestellte Frage verneint werden kann. In einem Filmmodul präsentiert das Unternehmen Jenapharm einen Film mit Ergebnissen einer Umfrage, in der Frauen ihre Meinung zu ihrer Periode äußern sollten. Die Daten suggerieren, dass die Menstruationsblutungen weitreichende Auswirkungen auf die Mehrheit der Frauen haben und/oder irgendwelche Probleme, negative Auswirkungen auf praktisch jeden Aspekt ihres Lebens haben (z. B. überdurchschnittlich hoher Verbrauch von Hygieneartikeln, Geschlechtsleben 95\%, Beeinträchtigung beruflicher Leistungsfähigkeit 80\%, Schwierigkeiten bei der Alltagsbewältigung 73\%, 70\% meiden gesellschaftliche Veranstaltungen, 36\% litten an starken Menstruationsblutungen). Der Sender präsentiert seine Produkte als eine Lösung dieser Probleme und als Hilfe, dank der die Kundinnen ihr Leben unbeschwerter genießen können.

Interessant ist in dem Pillen-Diskurs der Gebrauch des Begriffs Natürlichkeit, der im modernen Lifestyle eine wichtige Rolle spielt. Er wurde ursprünglich vor allem in Bezug auf die natürlichen Verhütungsmethoden verwendet. Gegenwärtig lässt sich ein Trend beobachten, den künstlichen (chemischen) Methoden die Eigenschaft der Natürlichkeit zuzusprechen und das Schlüsselwort Natürlichkeit hervorzuheben (siehe Abbildung Nr. 7). Es lässt sich beobachten, dass die Pharmakonzerne einerseits die „Natürlichkeit des Gebrauchs“ der Verhütungsmittel (die Pille ist etwas Alltägliches), auf der anderen Seite die Natürlichkeit des Produkts propagieren. Die Betonung der Natürlichkeit der hormonellen Verhütung ist eine Strategie, um die beworbenen Verhütungsmethoden aufzuwerten. Das Pharmaunternehmen Bayer wirbt beispielsweise

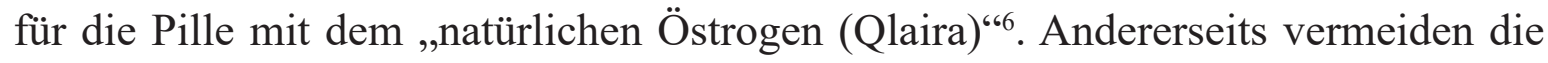
Pharmakonzerne die Bezeichnung der hormonellen Verhütung als künstlich, was früher praktiziert wurde. Die nicht hormonellen Methoden der Empfängnisverhütung werden dagegen auf den Webseiten über Verhütung zunehmend als alternative Methoden bezeichnet. Wir haben es also mit einer Strategie der Bedeutungsverschiebung zu tun.

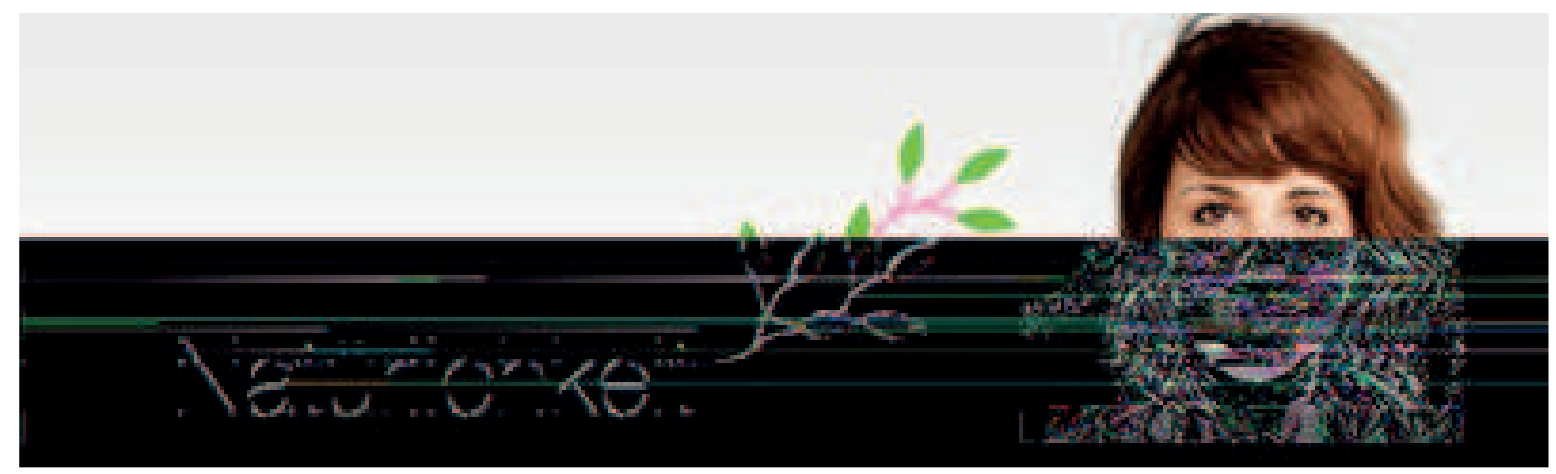

Abb. 7: Text-Cluster Natürlichkeit auf der Homepage von Jenapharm (www.jenapharm.de)

${ }^{6}$ Nina Bublitz (2009) stellt dazu fest, dass ,Natürlich“ in diesem Kontext weder sicherer noch besser verträglich bedeutet. Das Mittel enthält zwei Hormone: das Gestagen Dienogest, das so synthetisch ist wie alle anderen Pillen-Gestagene auch, und das Estradiolvalerat als Östrogen-Komponente. Estradiolvalerat wird im Körper in Estradiol umgewandelt; so gelangt man tatsächlich zum „,natürlichen Östrogen“ [...]. 
Es sei noch angemerkt, dass wenn die symptothermalen Methoden auf den Webseiten der Konzerne erwähnt werden, nicht ihre Vorteile, sondern ihre negativen Seiten betont werden und/oder vor diesen Methoden gewarnt wird.

c) Text-Cluster Pille und Sicherheit (Persuasive Handlung: Kopplung der Wirkungsweise hormoneller Verhütungsmittel mit Sicherheit)

Eine zentrale Bedeutung in den argumentativ orientierten Text-Clustern in dieser Gruppe hat das Sicherheitsargument. Der Sender verspricht, dass die angebotenen Mittel sicher sind und vor einer ungewollten Schwangerschaft, vor deren Konsequenzen und damit verbundenen Problemen in Familie und Beruf schützen können. Die Autoren der Webseiten suggerieren, dass mangelnde Sicherheit unterschiedliche Ängste zur Folge hat (u. a. Angst vor dem Vergessen der Einnahme der Pille, Angst vor unerwünschten Nebenwirkungen).

Die Pharmaunternehmen bewerben einerseits allgemein die Verhütungsmittel als unabdingbares Element des Sexuallebens und andererseits argumentieren sie, je nach Zielgruppe, für den Gebrauch eines konkreten Mittels, das sie produzieren. Es wird die Sicherheit der Produkte für jeden Frauentyp und jede Altersgruppe angepriesen. Bei jüngeren Frauen wird die Zuverlässigkeit und Sicherheit der Pille akzentuiert. Bei Frauen, die eher in stabilen Beziehungen leben, werden die „Unbequemlichkeit“ der Pille und Probleme mit ihrem Gebrauch (Probleme mit dem Vergessen der Pille oder nicht an sie denken wollen) genannt. Parallel wird die Strategie genutzt, Unsicherheitsgefühle in Bezug auf die Pille zu schüren. An diese Gruppe der Frauen werden als Alternative Informationen zu Langzeitverhütungsmethoden adressiert. An diesen Frauentyp sind Text-Cluster mit der Werbung für Langzeitverhütungsmethoden wie Hormonpflaster, Verhütungsring oder Hormonspirale gerichtet (siehe Abbildung Nr. 8). Das Bild zeigt eine zufriedene Frau, die ohne Stress ihr Leben genießt, weil sie sich für eine Langzeitverhütungsmethode entschieden hat.

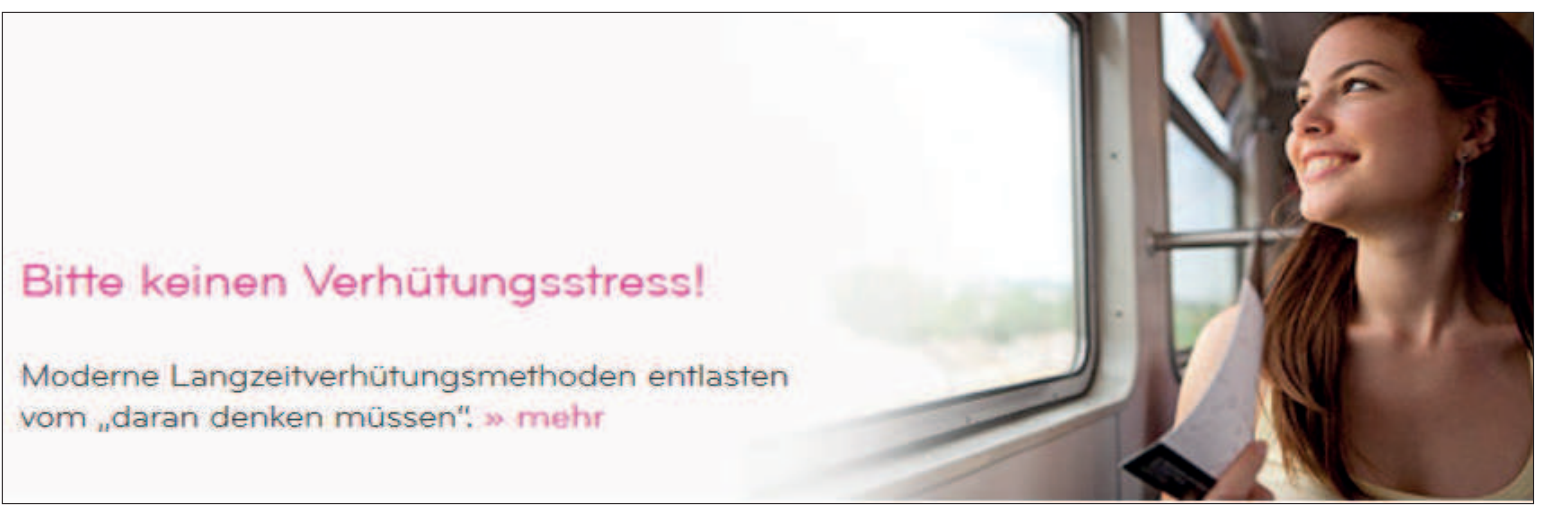

Abb. 8: Text-Cluster Bitte keinen Verhütungsstress! auf der Homepage von Jenapharm (www.meine-verhuetung.de) 
Der „flexible“ Lebensstil der Leserinnen wird präsupponiert und als Ursache von Ängsten, Zweifeln und belastenden Situationen im Leben junger Frauen identifiziert.

(12) Flexibilität als Lifestyle - ob im Job oder im Privatleben. Da scheint die regelmäßige Einnahme der Pille das kleinste Problem zu sein - bis ein Blick auf die Pillenpackung fällt und wir sehen, dass die Tablette vom Vortag noch in der Folie steckt. Und dann steigen Zweifel und Ängste hoch: Könnte ich nun schwanger sein? Bin ich jetzt noch zuverlässig mit der Pille geschützt? Diese Zweifel und Ängste können sehr belasten und sich negativ auf die Arbeit oder das Studium auswirken (msd, http://www.meine-verhütung.ch/Images/verhuten_zuverlassig.pdf).

Die Leserinnen werden auch mit Beispielen von Frauen konfrontiert, die vergessen haben, die Pille einzunehmen und jetzt besorgt sind und Angst vor einer ungeplanten Schwangerschaft haben. Der Sender verwendet das ad baculum Argument, indem er Beispiele präsentiert, in denen die Unsicherheit und Angst vor einer ungewollten Schwangerschaft bei Frauen und Männern negative Auswirkungen auf ihr Gefühls- und Beziehungsleben haben (msd, meine-verhütung.ch). Um das Ausmaß der Belastung zu illustrieren und um zu zeigen, wie die Partner mehr Sicherheit im Sexualleben haben können, gibt der Sender entsprechende Statistiken an, die von dem Bild eines besorgten Paares begleitet werden, das sich mit dem Rücken zugewandt ist.

Wussten Sie, dass...

- fast 50\% der Pillenanwenderinnen eine oder mehrere Pillen pro Zyklus vergessen?

- $82 \%$ der befragten Frauen bereits Situationen erlebt haben, in denen der Empfängnisschutz herabgesetzt war? Dies meist wegen vergessener Pillen.

- $65 \%$ der Schweizer Pillenanwenderinnen erleichtert sind, wenn bei ihnen die Menstruationsblutung eintritt?

- $77 \%$ der Pillenanwenderinnen nicht täglich an die Verhütung denken möchten? (msd, http://www.meine-verhütung.ch/Images/verhuten_zuverlassig.pdf).

Der Sender gebraucht auch die Autoritätsargumentation, um die Leserinnen von der Anwendung einer Langzeitverhütungsmethode zu überzeugen und Vertrauen zur neuen Methode zu gewinnen.
Gynäkologen bestätigen, dass Frauen oft Mühe haben, die Pille regelmäßig einzunehmen. Aus Gewohnheit bleiben viele bei der gewählten Verhütungsmethode. Wenn sie jedoch wechseln und eine Verhütungsmethode wählen, die nur wöchentlich, monatlich oder seltener angewendet wird, haben sie viel häufiger das Gefühl einer zuverlässigen Verhütung und das Vertrauen in ihr Verhütungsmittel steigt wieder (msd, http://www.meine-verhütung.ch/Images/verhuten_zuver- lassig.pdf).

Als Antonym zum Schlüsselwort Sicherheit wird Angst gebraucht. Die beabsichtigte Wirkung dabei ist, anzubieten, von Verhütungsmethoden Gebrauch zu machen, um Sexualität sorgenfrei genießen zu können und emotionale Belastung (aus mangelnder 
Sicherheit) zu vermeiden. Betont wird die Notwendigkeit der Verhütung, um die Ängste der Paare beim Sex zu eliminieren.

In den Text-Clustern aus dieser Gruppe suggerieren die Autoren, dass auch bei einem ungewollt ungeschützten Geschlechtsverkehr die Pharmaindustrie eine passende Lösung anzubieten hat und auch in so einem Fall Sicherheit garantieren kann. Der Gesichtsausdruck der Frau in Abbildung 9 zeigt, wie ungeschützter Sex zu bewerten ist.

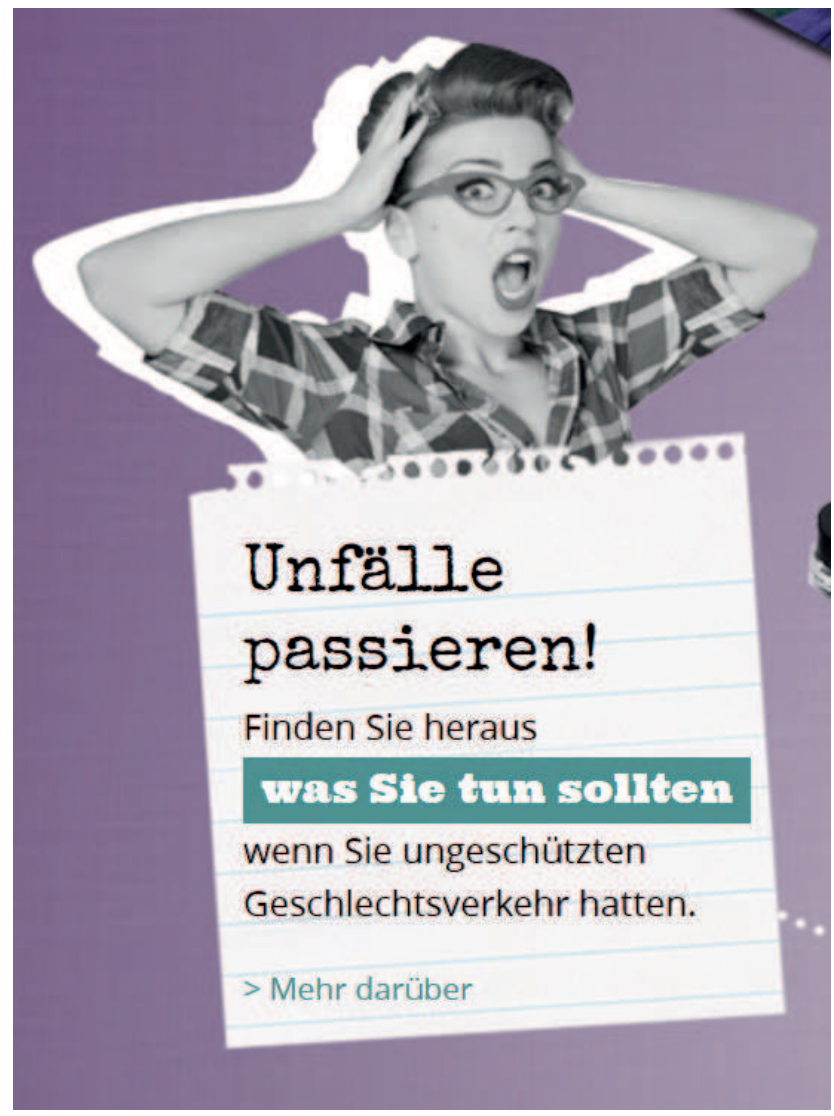

Abb. 9: Text-Cluster Unfälle passieren auf der Homepage von HRA Pharma (www.ellaone.at)

In diesen Text-Clustern finden wir außerdem persuasive Handlungen wie Modifikationen an Begriffen und Wendungen, die mit der Sicherheit der Pille zu tun haben und die auch typisch für den allgemeinen Pillen-Diskurs sind. Zu dieser Gruppe der Begriffsmodifikationen gehören metakommunikative Äußerungen des Senders zu Positionen, die laut ihm fälschlicherweise Teil des Diskurses sind. Diese Strategie könnte man als Imagearbeit für eigene Produkte bezeichnen. Dies kann mit einem Zitat von der Webseite der Firma Msd illustriert werden, in dem sie Stellung zu der Frage nimmt, ob die Pille nehmen ,sich mit Chemie vollstopfen bedeutet“". 
nachempfunden (msd, http://www.grunenthal.com/cms/cda/_common/inc/display_file. jsp?fileID=64000096).

Das Ziel solcher Modifikationen ist es, von eventuellen negativen Einstellungen zum Gebrauch der Pille abzubringen oder/und Korrekturen der Begriffe und Phrasen vorzunehmen. Im obigen Fall behauptet die Firma, der möglicherweise präsenten Meinung der Leserinnen zuwider, dass das Einnehmen der Verhütungspille kein sich mit Chemie vollstopfen sei. Die chemische Natur des Wirkstoffes wird jedoch nicht negiert. Die Leserinnen sollten nur den Eindruck bekommen, dass die Pilleneinnahme etwas mit einem natürlichen Prozess zu tun habe. Eine andere Bezeichnung für die Pille im Diskurs über Verhütung, die korrigiert wird, ist der Ausdruck Hormonbombe. Auf die Frage, ob die Pille danach eine Hormonbombe sei, antwortet der Hersteller (HRA Pharma):
Die Pille danach enthält zwar eine größere Menge an Hormonen als die normale Pille, jedoch wirken sie nur sehr kurz und gezielt. Die Pille danach ist gut verträglich. Die Pille danach un- terdrückt gezielt und einmalig den Hormonanstieg, der den Eisprung auslöst. Danach pendelt sich der Zyklus ganz von selbst wieder ein, man bekommt wieder einen Eisprung und kann auch schwanger werden, wenn man will (HRA Pharma, https://pille-danach.de/fragen/ist-die-pille-da- nach-eine-hormonbombe-659).

Die Pille danach wird hier indirekt nicht als Hormonbombe anerkannt. Dafür werden positive Aspekte (die kurze, einmalige und gezielte Wirkung, Verträglichkeit, Rückkehr zum normalen Zustand, Möglichkeit problemloser Schwangerschaft nach der Behandlung) genannt, um die Vorteile der Pille danach in den Vordergrund zu rücken. Verwandt mit obigen Modifikationen sind metakommunikative Äußerungen der Pharmaunternehmen über die Richtigkeit bestimmter diskursiver Positionen. In der Opposition zu den korrigierenden Handlungen in obigen Beispielen gibt es Module, in denen die Unternehmen positiv formulierte und zugleich ihre Position verstärkende Behauptungen aufstellen. Das Modul Fakten, die richtig sind der Firma Msd ist ein Beispiel für Module, in denen der Sender den Anspruch erhebt zu definieren, was wahr ist.

\footnotetext{
Wahr ist, dass nach Absetzen der Pille auch nach jahrelanger Pilleneinnahme sofort wieder eine Schwangerschaft möglich ist (HRA Pharma, https://pille-danach.de/fragen/ist-die-pille-danach-eine-hormonbombe-659).
}

Eine explizite Formulierung dessen, was wahr ist, sollte eine klare persuasive Wirkung bei den Leserinnen erzielen und sie davon überzeugen, dass die Pille sicher ist. Als Begründung der These in der umstrittenen Frage, ob man nach jahrelanger Einnahme der Pille schwanger werden kann, wird das modale Adjektiv möglich gebraucht. Durch das explizite Definieren des Wahren und den Kontext anderer Äußerungen in dem Modul, tritt die alethische Modalität des Satzes in den Hintergrund. Den Leserinnen 
wird suggeriert, dass eine Schwangerschaft in solchen Fällen ohne Probleme erfolgen kann. Die Sicherheit der Pille wird auch in dieser Hinsicht beteuert.

Als eine manipulative Maßnahme in der Vermittlung der Informationen über Verhütung in den untersuchten Text-Clustern in dieser Gruppe kann man das Auslassen, Meiden oder das Minimieren der Nebenwirkungen der beworbenen Produkte bezeichnen. Es wird sehr allgemein über die Nebenwirkungen berichtet.

Nebenwirkungen sind individuell und treten bei jedem unterschiedlich stark oder auch gar nicht auf. Die Nebenwirkungen der Pille danach sind im Normalfall mild und klingen von selbst wieder ab (HRA Pharma http://www.die-pille-danach.at/fragen-antworten/nebenwirkungen).

Eine andere Variante, um das Thema Nebenwirkungen zu behandeln, ist es, sie nicht anzusprechen. Der Autor verweist dagegen explizit auf die Frauenärztin bzw. den Frauenarzt, von denen die Kundinnen Näheres zu diesem Thema erfahren sollten. Auf der Seite langzeitverhuetung.com des Herstellers Jenapharm ist folgender Text in Bezug auf alle dort genannten Verhütungsmethoden (Verhütungsschirmchen, Kupferspirale, Kupferkette, Hormonimplantat und Pille) zu sehen:

(19) Wie immer, wenn ein Arzneimittel angewendet wird, können Nebenwirkungen auftreten. Bitte sprich, wenn Du Fragen zu Nebenwirkungen hast, mit Deiner Frauenärztin bzw. Deinem Frauenarzt (Jenapharm, langzeitverhuetung.com).

Dadurch werden keine konkreten Nebenwirkungen der Verhütungsmittel genannt und bei den Leserinnen entstehen somit keine negativen Assoziationen mit den beworbenen Methoden der Verhütung. Wenn die negativen Nebenwirkungen erwähnt werden, werden neben ihnen, um ein besseres Bild der Präparate zu vermitteln, auch die positiven Effekte genannt, die als ,günstig“ bezeichnet werden. Auf der Webseite pille-fuer-mich.de lautet der Titel einer der Rubriken „Stabiler Zyklus, reine Haut, günstige Nebenwirkungen" (pille-fuer-mich.de). Ähnliche Beispiele findet man auch bei gynaekologie.de oder Hexal.de.

\section{Fazit}

Die Adressaten der persuasiven Botschaften in den Text-Clustern sind vor allem junge Frauen, die eine sehr wichtige Zielgruppe für den Absatz der Pharmaprodukte darstellen. Sie bilden auch eine Öffentlichkeit, die aus der Initiative des Senders aktiv an dem von den Pharmafirmen betriebenen Verhütungs-Diskurs teilnimmt und die Positionen und Einstellungen der Pharmaunternehmen in Form von verschiedenen Dialogmöglichkeiten bestätigt.

Mittels unterschiedlicher Text-Cluster gestalten Pharmaunternehmen ihre persuasiven Botschaften. Die Analyse hat gezeigt, dass Pharmaunternehmen mit ihren per- 
suasiven Botschaften nicht nur für ihre Produkte, für die Verhütungsmittel, sondern auch für den damit verbundenen Lebensstil, Lifestyle, werben, um die Einstellung der Empfängerinnen zur Sexualität zu beeinflussen. Eine besondere persuasive Rolle spielen hier Text-Cluster mit Bedeutungsmodifikationen, in denen auch neue Assoziationen gebildet werden und schon existierende, dem Zeitgeist entsprechende Begriffe wie Liebe verstärkt werden. Die Pharmaunternehmen betonen die Unabhängigkeit, die Selbstbestimmung und die Freiheit der Leserinnen und somit ihr Recht auf freie Entscheidung. Repräsentativ für diese Position ist das Motto ,Verhütung. Meine Sache“ (Jenapharm). Den Nutzerinnen wird visuell und sprachlich nahegelegt, dass sie durch den Gebrauch der Pille und anderer Mittel ihre Freiheit ausleben und unabhängig bleiben können. Zufriedene und glückliche Frauen, Mädchen und Paare erfüllen die Rolle der visuellen Beweise für die Wirkungsweise der hormonellen Präparate. Bilder verzweifelter und besorgter Frauen und Paare zeigen die Konsequenzen, wenn man nicht an Verhütung denkt und die beworbenen Produkte nicht in Anspruch nimmt.

Über die stilistische Ausgestaltung der Webseiten werden konkrete Frauenbilder, Werte, diskursive Positionen der Autoren der Webseiten (z. B. die Einstellung zur Sexualität) vermittelt. Die Schwangerschaft wird als etwas Negatives, als Bedrohung für die Freiheit und Unabhängigkeit betrachtet. Sex zu haben wird zwangsläufig mit Verhütung gekoppelt. Es wird suggeriert, dass wenn jemand Sex haben will, er automatisch an Verhütung denken muss. Verhütung wird dabei als ein natürliches Phänomen definiert, dass seit der Steinzeit existiert. Die Frau sollte nicht überlegen, ob sie eine Verhütung braucht, sondern nur welche Pille oder welche angebotene Verhütungsmethode am besten zu ihr passt.

\section{Literatur}

Blech, J. (2013). Medikamente. Zähes Blut. Der Spiegel, 6, 114.

Bublitz, N. (2009). Wie Frauen mit „Natürlichkeit“ gelockt werden. Stern 08. Oktober 2009. Abgerufen von http://www.stern.de/gesundheit/marketing-fuer-antibabypille-qlaira-wie-frauen-mit--natuerlichkeit--gelockt-werden-3445944.html [Zugriff: 20.11.2015]

Diekwisch, H. \& Schaaber, J. (2009). Direktwerbung für rezeptpflichtige Arzneimittel - Verbraucheraufklärung oder Beitrag zur Medikalisierung? Zeitschrift für Allgemeine Medizin Deutscher Ärzte-Verlag, 85 (8), 329-334.

Duden, B. (1996). Von ,der “ Pille und unserem „Zustand“. In G. Staupe \& L. Vieth (Hrsg.), Die Pille: Von der Lust und von der Liebe (S. 67-79). Berlin: Rowohlt.

Fix, U. (1996). Textstile und KonTextstile. Stil in der Kommunikation als umfassende Semiose von Sprachlichem, Parasprachlichem und Außersprachlichem. In U. Fix \& G. Lerchner (Hrsg.), Stil und Stilwandel (S. 111-132). Frankfurt am Main: Peter Lang.

Herbig, A \& Sandig, B. (1994). Das kann doch wohl nur ein Witz sein! Argumentieren, Bewerten und Emotionalisieren im Rahmen persuasiver Strategien. In M. Moilanen (Hrsg.), Überredung in der Presse (S. 59-98). Berlin: de Gruyter.

Karmasin, H. (2001). Die geheime Botschaft unserer Speisen. Was Essen über uns aussagt. Bergisch Gladbach: Bastei Lübbe. 
Klug, N.-M. \& Stöckl, H. (Hrsg.). (2016). Handbuch Sprache im multimodalen Kontext. Berlin-Boston: de Gruyter.

Kravitz, R. \& Epstein, R. et al. (27 April 2005). Influence of patients' requests for direct-to-consumer advertised antidepressants: a randomized controlled trial. Journal of the American Medical Association (JAMA), 293 (16), 1995-2002.

Püschel, U. (1997). Puzzle-Texte - Bemerkungen zum Textbegriff. In G. Antos \& H. Tietz (Hrsg.), Die Zukunft der Textlinguistik. Traditionen, Transformationen, Trends (S. 27-41). Tübingen: de Gruyter.

Rolf, E. (1997). Illokutionäre Kräfte. Grundbegriffe der Illokutionslogik. Opladen: Westdeutscher Verlag.

Schindele, E. (2012). Wie die Pille die Selbstwahrnehmung verändert(e) - persönliche Erfahrungen und philosophische Überlegungen. Frauengesundheit-Generationen im Dialog: Dokumentation der 19. AKF-Jahrestagung 2012. Abgerufen von http://www.akf-info.de/portal/wp-content/uploads/2015/07/6_ Schindele.pdf [Zugriff: 10.09.2015]

Staupe, G. \& Vieth, L. (Hrsg.). (1996). Die Pille - Von der Lust und von der Liebe. Berlin: Rowohlt.

\section{Internetquellen}

Bundeszentrale für gesundheitliche Aufklärung: Verhütungsverhalten Erwachsener - Ergebnisse der Repräsentativbefragung, Köln 2011. Abgerufen von http://www.forschung.sexualaufklaerung.de/ fileadmin/fileadmin-forschung/pdf/BZGA-1100988_Verhue_tungsverhalten_Erwachsener_DE_low. pdf [Zugriff: 20.12.2015]

Bundeszentrale für gesundheitliche Aufklärung: Jugendsexualität im Internetzeitalter, Köln 2013. Abgerufen von https://publikationen.sexualaufklaerung.de/index.php?docid=2894 [Zugriff: 20.12.2015] 